\title{
A VARIANCE METHOD IN COMBINATORIAL NUMBER THEORY
}

\author{
by IAN ANDERSON
}

(Received 30 April, 1968)

Let $s=s\left(a_{1}, a_{2}, \ldots, a_{r}\right)$ denote the number of integer solutions of the equation

$$
u_{1}+u_{2}+\ldots+u_{r}=\left[\frac{1}{2} \sum_{i=1}^{r} a_{i}\right]=\lambda
$$

subject to the conditions

$$
0 \leqq u_{i} \leqq a_{i} \quad(i=1, \ldots, r)
$$

the $a_{i}$ being given positive integers, and square brackets denoting the integral part. Clearly $s\left(a_{1}, \ldots, a_{r}\right)$ is also the number $s=s(m)$ of divisors of $m=p_{1}^{a_{1}} p_{2}^{a_{2}} \ldots p_{r}^{a_{r}}$ which contain exactly $\lambda$ prime factors counted according to multiplicity, and is therefore, as is proved in [1], the cardinality of the largest possible set of divisors of $m$, no one of which divides another.

In an earlier paper [2] we proved by means of contour integration that, under fairly general conditions,

$$
s(m) \sim \sqrt{\left(\frac{2}{\pi}\right)} \frac{\tau(m)}{\sqrt{ } A(m)}
$$

as $\sum_{i=1}^{r} a_{i} \rightarrow \infty$, where $\tau(m)=\prod_{i=1}^{r}\left(1+a_{i}\right)$ denotes the total number of divisors of $m$, and where $A(m)=\frac{1}{3} \sum_{i=1}^{r} a_{i}\left(a_{i}+2\right) . \quad$ In the squarefree case, when $m=p_{1} p_{2} \ldots p_{n}$, this becomes

$$
\left(\begin{array}{c}
n \\
{\left[\frac{1}{2} n\right]}
\end{array}\right) \sim \sqrt{\left(\frac{2}{\pi}\right)} \frac{2^{n}}{\sqrt{ } n}
$$

a result which checks with Stirling's formula. We now prove

THEOREM 1. There exist constants $C_{1}>0, C_{2}>0$ such that

for all $m$.

$$
C_{1} \frac{\tau(m)}{\sqrt{ } A(m)} \leqq s(m) \leqq C_{2} \frac{\tau(m)}{\sqrt{A(m)}}
$$

If we define the degree $n$ of an integer $m$ to be the number of prime factors of $m$ counted according to multiplicity, and let $N_{l}=N_{l}(m)$ denote the number of divisors of $m$ of degree $l$, we then have $s=N_{\lambda}$, where $\lambda=\left[\frac{1}{2} n\right]$. Also let $\tau=\tau(m)$.

Let

$$
\sigma^{2}=\frac{2}{\tau} \sum_{l=0}^{\lambda}\left(\frac{n}{2}-l\right)^{2} N_{l}=\frac{1}{2} \tau_{l=0}^{\lambda}(n-2 l)^{2} N_{l}
$$


denote the variance of the distribution of degrees among the divisors of $m$. Since the variance of $\{0,1,2, \ldots, a\}$ is $\frac{1}{12} a(a+2)$, and since the variance of a sum of independent distributions is equal to the sum of the variances, we have

$$
\sigma^{2}=\sum_{i=1}^{r} \frac{1}{12} a_{i}\left(a_{i}+2\right)=\frac{1}{4} A(m) .
$$

To prove Theorem 1, it therefore suffices to prove

THEOREM 2. With the above notation, there exist constants $C_{3}>0, C_{4}>0$ such that

$$
C_{3} \frac{\tau}{s} \leqq \sigma \leqq C_{4} \frac{\tau}{s}
$$

2. The lower bound. Since

we have

$$
\left(\begin{array}{c}
x+1 \\
3
\end{array}\right)-\left(\begin{array}{c}
x-1 \\
3
\end{array}\right)=(x-1)^{2},
$$

$$
\begin{aligned}
2 \tau \sigma^{2} & =\sum_{l=0}^{\lambda}(n-2 l)^{2} N_{l}>\sum_{l=0}^{\lambda}(n-2 l-1)^{2} N_{l} \\
& =\sum_{l=0}^{\lambda}\left\{\left(\begin{array}{c}
n+1-2 l \\
3
\end{array}\right)-\left(\begin{array}{c}
n-1-2 l \\
3
\end{array}\right)\right\} N_{l} \\
& =\sum_{l=1}^{\lambda}\left(\begin{array}{c}
n+1-2 l \\
3
\end{array}\right)\left(N_{l}-N_{l-1}\right)+\left(\begin{array}{c}
n+1 \\
3
\end{array}\right) .
\end{aligned}
$$

Now de Bruijn, Tengbergen and Kruyswijk [1] have shown that the divisors of $m$ can be put into $s$ disjoint chains ordered by divisibility, the number of chains containing $n+1-2 l$ elements being precisely $N_{l}-N_{l-1}$. (Incidentally, the result

$$
N_{\iota} \leqq N_{l+1} \quad \text { if } \quad l<\lambda
$$

is implicit in this.) Thus $2 \tau \sigma^{2}$ is essentially just the number of ways of selecting three divisors of $m$ from the same chain.

If the $s$ chains contain $x_{1}, x_{2}, \ldots, x_{s}$ elements, where $\sum_{i=1}^{s} x_{i}=\tau$, we have

$$
2 \tau \sigma^{2}>\frac{1}{6} \sum_{i=1}^{s} x_{i}\left(x_{i}-1\right)\left(x_{i}-2\right) .
$$

This has a minimum if all the $x_{i}$ are equal, when $x_{i}=\tau / s$. Thus, for sufficiently large $n$,

$$
2 \tau \sigma^{2} \geqq\left(\frac{1}{6}-\varepsilon\right) \frac{\tau^{3}}{s^{2}},
$$

since $s=o(\tau)$ (see [2]; alternatively, this follows from the second half of this theorem). 
Thus

$$
\sigma \geqq\left(\frac{1}{2 \sqrt{3}}-\varepsilon\right) \frac{\tau}{s}
$$

3. The upper bound. In view of (1), we have to estimate $\sum_{l=0}^{\lambda}(n-2 l)^{2} N_{l}$. To do this, we make use of the following lemmas, the first of which is proved by an elementary argument in [3].

LEMma 1. If $0<l<k<n$, then $N_{l} N_{k} \leqq N_{l+1} N_{k-1}$.

Lemma 2. (Reduction Formula)

$$
\begin{aligned}
N_{\lambda-r}^{2} \sum_{l=0}^{\lambda-r}(n-2 l)^{2} N_{l} \leqq N_{\lambda-r-1}^{2} \sum_{l=0}^{\lambda-r-1}(n-2 l)^{2} N_{l}+8 N_{\lambda-r-1}^{2} \sum_{l=0}^{\lambda-r-1}(n-2 l) N_{l} & +16 N_{\lambda-r-1}^{2} \sum_{l=0}^{\lambda-r-1} N_{l}+3(2 r+5)^{2} N_{\lambda-r .}^{3} .
\end{aligned}
$$

Proof. Throughout, we shall make repeated use of (2).

$$
N_{\lambda-r}^{2} \sum_{l=0}^{\lambda-r}(n-2 l)^{2} N_{l} \leqq N_{\lambda-r}^{2} \sum_{l=0}^{\lambda-r-2}(n-2 l)^{2} N_{l}+2(2 r+1)^{2} N_{\lambda-r}^{3},
$$

where the first term on the right is, by two applications of Lemma 1,

$\leqq N_{\lambda-r-1}^{2} \sum_{l=0}^{\lambda-r-2}(n-2 l)^{2} N_{l+2}$

$\leqq N_{\lambda-r-1}^{2} \sum_{l=0}^{\lambda-r}(n-2 l+4)^{2} N_{l}$

$\leqq N_{\lambda-r-1}^{2} \sum_{l=0}^{\lambda-r-1}(n-2 l+4)^{2} N_{l}+(2 r+5)^{2} N_{\lambda-r}^{3}$

$\leqq N_{\lambda-r-1}^{2} \sum_{l=0}^{\lambda-r-1}(n-2 l)^{2} N_{l}+8 N_{\lambda-r-1}^{2} \sum_{l=0}^{\lambda-r-1}(n-2 l) N_{l}+16 N_{\lambda-r-1}^{2} \sum_{l=0}^{\lambda-r-1} N_{l}+(2 r+5)^{2} N_{\lambda-r}^{3}$.

The lemma now follows.

Starting with $r=0$, we apply this reduction formula repeatedly to obtain

$$
\begin{aligned}
2 \tau s^{2} \sigma^{2} & =N_{\lambda}^{2} \sum_{l=0}^{\lambda}(n-2 l)^{2} N_{l} \\
& \leqq 8 \sum_{t=0}^{\lambda-1} N_{\lambda-t}^{2}\left(\sum_{l=0}^{\lambda-t}(n-2 l) N_{l}\right)+16 \sum_{t=0}^{\lambda-1} N_{\lambda-t}^{2}\left(\sum_{l=0}^{\lambda-t} N_{l}\right)+3 \sum_{t=0}^{\lambda}(2 t+5)^{2} N_{\lambda-t}^{3} .
\end{aligned}
$$


But

$$
\begin{aligned}
\sum_{t=0}^{\lambda}(2 t+5)^{2} N_{\lambda-t}^{3} & \ll \sum_{t=0}^{\lambda} N_{\lambda-t}\left(t N_{\lambda-t}\right)^{2} \\
& \ll \sum_{t=0}^{\lambda} N_{\lambda-t}\left(N_{\lambda-t}+\ldots+N_{\lambda-1}\right)^{2} \\
& \ll\left(\frac{\tau}{2}\right)^{2} \cdot \frac{\tau}{2} \ll \tau^{3},
\end{aligned}
$$

and

$$
\sum_{t=0}^{\lambda-1} N_{\lambda-t}^{2}\left(\sum_{l=0}^{\lambda-t} N_{l}\right) \leqq \frac{\tau}{2} \sum_{t=0}^{\lambda-1} N_{\lambda-t}^{2} \leqq \frac{\tau}{2}\left(\sum_{t=0}^{\lambda-1} N_{\lambda-t}\right)^{2} \ll \tau^{3}
$$

Further, a reduction argument similar to the above [3] shows that

$$
N_{\lambda} \sum_{l=0}^{\lambda}(n-2 l) N_{l} \ll \tau^{2}
$$

Thus the remaining term in (3) is

$$
\ll \tau^{2} \sum_{t=0}^{\lambda-1} N_{\lambda-t} \ll \tau^{3}
$$

Thus finally

$$
2 \tau s^{2} \sigma^{2} \ll \tau^{3}
$$

whence

$$
\sigma \ll \frac{\tau}{s} .
$$

This completes the proof of Theorem 2. We remark, however, that a more careful estimation gives the result

$$
\sigma \leqq \frac{\sqrt{11} \tau}{2} \frac{1}{s}
$$

provided the degree of $m$ is big enough. Whereas the lower bound is the best possible, being attained when $m$ is a prime power, our upper bound can certainly be improved upon. Perhaps its value in the case of $m$ squarefree, namely $1 / \sqrt{ }(2 \pi)$, is the true value.

\section{REFERENCES}

1. N. G. de Bruijn, C. van E. Tengbergen and D. Kruyswijk, On the set of divisors of a number Nieuw Arch. Wiskunde (2) 23 (1951), 191-193.

2. I. Anderson, On primitive sequences, J. London Math. Soc. 42 (1967), 137-148.

3. I. Anderson, On the divisors of a number, J. London Math. Soc. 43 (1968), 410-418.

\section{UNIVERSITY OF GLASGOW}

